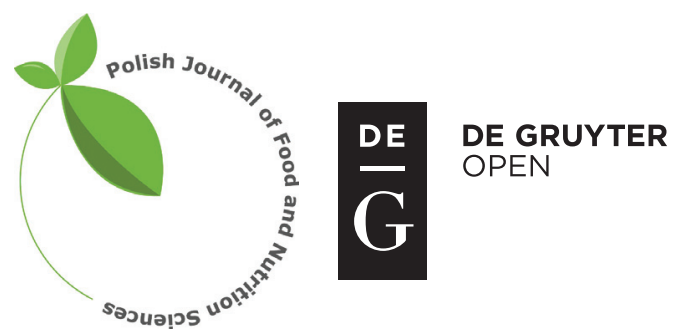

Pol. J. Food Nutr. Sci., 2017, Vol. 67, No. 4, pp. 327-337

DOI: $10.1515 /$ pjfns-2017-0003 http://journal.pan.olsztyn.pl

Original research article

Section: Nutritional Research

\title{
Evaluation of High Fibers Okara and Soybean Bran as Functional Supplements for Mice with Experimentally Induced Type 2 Diabetes
}

\author{
Manal Ismaiel ${ }^{1}$, Hong Yang ${ }^{1,2,3,4,5, *}$, Min Cui $^{6,7}$ \\ ${ }^{I}$ College of Food Science and Technology, Huazhong Agricultural University, Wuhan, Hubei 430070, China \\ ${ }^{2}$ Collaborative Innovation Center for Efficient and Health Production of Fisheries in Hunan, \\ Changde, Hunan 415000, China \\ ${ }^{3}$ Key Laboratory of Environment Correlative Dietology, Huazhong Agricultural University, Ministry of Education, \\ Wuhan, Hubei 430070, China \\ ${ }^{4}$ National R\&D Branch Center for Conventional Freshwater Fish Processing (Wuhan), Wuhan, Hubei 430070, China \\ ${ }_{5}^{5}$ Aquatic Product Engineering and Technology Research Center of Hubei Province, Wuhan, Hubei 430070, China \\ ${ }^{6}$ State Key Laboratory of Agricultural Microbiology, Huazhong Agricultural University, Wuhan, Hubei 430070, China \\ ${ }^{7}$ Laboratory of Animal Virology, College of Veterinary Medicine, Huazhong Agricultural University, \\ Wuhan, Hubei 430070, China Phone: 86-27-87280170,Fax: 86-27-87278373, E-mail: cuimin@mail.hzau.edu.cn
}

Key words: okara, soybean bran, type 2 diabetes, hyperglycemia, hypolipidemic effect

The objective of this study was to evaluate and compare the anti-diabetic efficacy of feeding diets supplemented with okara and soybean bran to ICR mice with experimentally-induced type 2 diabetes. While okara and soybean bran are from the same source, there is no performed research comparing the effects of these soybean byproducts on glycemic status. Normal and streptozotocin-induced type 2 diabetic ICR mice were assigned either to a normal diet in the normal control group, a high fat diet only in the diabetic control group, a high fat diet supplemented with $15 \%$ okara in the okara group, a high fat diet supplemented with $15 \%$ soybean bran in the soybean bran group or a high-fat diet supplemented with $0.1 \%$ metformin in the metformin group for 8 weeks. The biochemical parameters, the organs relative weights and liver histological structure of mice were determined. Okara was significantly effective in controlling hyperglycemia and improving glucose tolerance. Moreover, the antihyperglycemic effect of okara was broadly comparable with the actions of metformin. Feeding okara and soybean bran caused hypolipidemic effects. In addition, they had a strong cytoprotective effect on hepatocytes. Soybean bran seemed more efficient than okara in alleviating hepatic cell histological changes. Results demonstrated the potential benefit of okara and soybean bran in glycemic control and reducing the risk of type 2 diabetes complications.

\section{INTRODUCTION}

Type 2 diabetes mellitus (T2DM) is a disease caused by relative lack of insulin or a loss of insulin function in various organs, resulting in many clinical symptoms as well as malfunctioning metabolism in carbohydrate, lipid and protein [Murotomi et al., 2015]. Dietary interference is one of the most important therapies suggested for the patient with T2DM [Tucker et al., 2014]. The target of the diet therapy is not only to reduce body weight, but also to achieve a beneficial role against the major defects of T2DM, such as insulin resistance, hyperglycemia and hyperlipidemia [Cicek et al., 2009]. High-fiber diets from a variety of foods could reduce fasting plasma glucose [Kiehm et al., 1976], cholesterol and triacylglycerol levels [Anderson \& Ward, 1979]. The glucose tolerance could be significantly improved by the addi-

\footnotetext{
* Corresponding Author: Tel.: 86-27-87672111; Fax: 86-27-87278373;

E-mail: yangh@mail.hzau.edu.cn (Hong Yang)
}

tion of dietary fiber source into the test meals of both healthy and diabetic subjects [Mahalko et al., 1984]. Moreover, a considerable reduction was observed in postprandial glucose and insulin level in rats after two-week feeding of guar or carboxymethyl cellulose [Cannon et al., 1980]. Dietary fiber could reduce the rate of diffusion of digestion products towards the absorptive mucosal surface and enhance the release of gastrointestinal hormones affecting glucose metabolism [Madar, 1983].

Soybean-based foods have well-documented beneficial effects on human health and currently the consumption of soybean products elevated due to functional food improving knowledge [Dueñas et al., 2012]. As a good source of dietary fiber, soybean bran is a major co-product of the soybean processing industry and made from soybean hull (seed coat of soybeans). It constitutes about $8 \%$ of the whole seed and contains about $86 \%$ complex carbohydrates. Soy hull, as a source of dietary fiber, could increase the plasma high density lipoprotein cholesterol of type 2 diabetic subjects [Mahalko et al., 1984]. 
Okara is the insoluble residue of the production of soy milk from soybean [Yogo et al., 2011] and mainly rich in dietary fiber $(50-60 \%)$ and protein (30\%) [Préstamo et al., 2007]. Its dietary fiber has $12 \%$ hemicellulose, $5.6 \%$ cellulose, $12 \%$ lignin, and $0.16 \%$ phytic acid [Surel \& Couplet, 2005]. Because of its high fiber content, okara can be used as a supplement in human diets, particularly western diets, which are deficient of the essential fiber [Préstamo et al., 2007]. There were only a limited number of studies on antidiabetic effect of okara [Ahmad et al., 2010; Lemes et al., 2014]. They indicated that the okara diet might be of great beneficial effects on glycemic control, preventing glucose intolerance and reducing the risk of diabetic complications, such as hypercholesterolemia.

While okara and soybean bran are from the same source, there is no performed research comparing the effects of these soybean byproduct on glycemic status. Therefore, comparison study is needed to clarify the differences in their antidiabetic effects in order to develop suitable foods for type 2 diabetic patients. The objective of this study was to evaluate and compare the anti-diabetic efficacy of feeding diets supplemented with soybean bran or okara to ICR mice with experimentally-induced type 2 diabetes.

\section{MATERIALS AND METHODS}

\section{Diet preparation}

Fresh okara was obtained from a tofu production supplier at Huazhong Agricultural University (Wuhan, Hubei, China). Firstly, okara was dried in an air blowing thermostatic oven (DHG-9240A, Jinghong Experiment Equipment Industry Co., Ltd., Shanghai, China) at $60^{\circ} \mathrm{C}$ for $24 \mathrm{~h}$ with $3 \mathrm{~h}$ interval stirring. Then it was ground to fine powder using a $200 \mathrm{~g}$ portable grinder (Lin Da Machinery Ltd. of Wenling, Zhejiang, China) for $3 \mathrm{~min}$. Ground okara was sieved through an
80 mesh sieve and stored in polyethylene bags. Soybean bran was ground and sieved as the above method. The preparation of experimental diets was done by adding $15 \%$ of okara, $15 \%$ of soybean bran or $0.1 \%$ of metformin, metformin hydrochloride (Beijing Zhonghui Pharmaceutical Co., Beijing, China) into the high-fat diet (HFD). Table 1 includes the compositions of normal, high-fat and experimental diets.

\section{Animals and experimental design}

A total number of 60 ICR male mice (three-week old and 18-20 g body weight) were obtained from Hunan Slack Laboratory Animals Co., Ltd. (Changsha, Hunan, China) and kept in a temperature- and humidity-controlled facility. Diets and tap water were provided ad libitum. All procedures were performed in accordance with the guidelines on the ethical use of animals issued by the Animal Care and Use Committee of Huazhong Agricultural University. After all mice were fed the normal diet (Table 1) within one week of habituation to the study environment, 10 mice were selected randomly and received continuous feeding of a normal diet performed as a normal control group (NCG). In order to develop the diabetic animal model, the remaining mice were fed with highfat diet (HFD) for additional six weeks (4 weeks before STZ injection and 2 weeks after injection). Diabetes was induced by an intraperitoneal injection of five daily consecutive multiple low doses of freshly prepared streptozotocin (STZ) $(40 \mathrm{mg} / \mathrm{kg}$ body weight, Aladdin Biological Technology, Co., Ltd. Shanghai, China) dissolved in $0.1 \mathrm{~mol} / \mathrm{L}$ citrate buffer ( $\mathrm{pH} 4.4)$ [Jin et al., 2014]. The animals with fasting blood glucose (FBG) level $>7.0 \mathrm{mmol} / \mathrm{L}$ were considered as T2DM.

\section{Experimental groups}

The experimentally-induced type 2 diabetic mice were divided into four groups, 10 mice in each. Mice in the first group con-

TABLE 1. Composition of the normal diet, high-fat diet, okara diet, soybean bran diet, and metformin diet.

\begin{tabular}{|c|c|c|c|c|c|}
\hline Component & Normal diet (\%) & High-fat diet (\%) & Okara diet $(\%)$ & Soybean diet (\%) & Metformin diet (\%) \\
\hline Corn meal & 57.7 & 50.5 & 48 & 48 & 50.4 \\
\hline Wheat bran & 17.0 & 10.0 & 5.0 & 5.0 & 10.0 \\
\hline Soybean meal & 18.0 & 10.0 & 3.0 & 3.0 & 10.0 \\
\hline Fish meal & 6.0 & 3.0 & 3.0 & 3.0 & 3.0 \\
\hline Egg yolk powder & 1.0 & 5.0 & 4.5 & 4.5 & 5.0 \\
\hline Vitamin mixture & 0.1 & 0.1 & 0.1 & 0.1 & 0.1 \\
\hline Trace elements & 0.2 & 0.2 & 0.2 & 0.2 & 0.2 \\
\hline Cholesterol & - & 1.0 & 1.0 & 1.0 & 1.0 \\
\hline Lard & - & 10 & 10 & 10 & 10 \\
\hline Sucrose & - & 10 & 10 & 10 & 10 \\
\hline Sodium cholate & - & 0.2 & 0.2 & 0.2 & 0.2 \\
\hline Okara & - & - & 15 & - & - \\
\hline Soybean bran & - & - & - & 15 & - \\
\hline Metformin & & - & - & - & 0.1 \\
\hline
\end{tabular}


tinued feeding with HFD which served as the diabetic control group (DCG). The second group was fed HFD supplemented with $15 \%$ okara (HF-OKG). The third group was fed with HFD supplemented with $15 \%$ soybean bran (HF-SBG). The fourth group was fed with HFD supplemented with $0.1 \%$ metformin (HF-MEG). All mice were maintained on their own diet for another eight weeks. The high-fat diet supplemented with metformin was kept at $4^{\circ} \mathrm{C}$ to make sure the stability of drug.

\section{Measurements of food intake, water consumption and body weight}

Daily fluid and food intake were measured during the entire experimental period. The mouse body weight was recorded weekly by an electronic balance, while the food and water intake were calculated every day by subtracting the remaining amounts from the one supplied at the same time during the experimental period.

\section{Fasting blood glucose}

Fasting blood glucose level of each mouse from different groups was measured weekly after overnight $12-\mathrm{h}$ fasting period. The blood sample was collected after a cut was made on the tail. Blood glucose level was determined using an AccuChek Active blood glucose meter and active test strips (Roche Diagnostic GmbH, Mannheim, Germany).

\section{Oral glucose tolerance test (OGTT)}

The OGTT was conducted at week 7 of the experiment period. The mice in all groups were weighed and fasted from the feed for $12 \mathrm{~h}$. D-glucose solution (50\%, $2 \mathrm{~g} / \mathrm{kg}$ body weight) was administrated orally to fasting mice using a 20-gauge stainless steel gavages feeding needle. The blood sample was collected from a tail-cut, while the blood glucose levels were measured at time $0,30,60$, and 120 min after glucose administration.

\section{Insulin tolerance test (ITT)}

The ITT was performed at week 7. After 4-h fasting, insulin $(0.375 \mathrm{IU} / \mathrm{kg}$, NovoRapid 30 asparaginyl, Novo Nordisk Company, Denmark) was dosed to the mice at $5 \mathrm{~mL} / \mathrm{kg}$ via subcutaneous injection. The blood-glucose levels were measured by collecting blood samples from the tail-cut after insulin injection $(0,30,60$, and $120 \mathrm{~min})$.

\section{Animal sacrifice}

At the end of the study period, mice were fasted overnight for $12 \mathrm{~h}$ and then sacrificed. The eyeballs of the mice were removed [Xu et al., 2015] and blood was drawn. After bleeding, mice were euthanized by cervical decapitation. The samples of liver, kidney, heart, spleen, pancreas and epididymal fat were washed with $0.85 \%$ cold saline, dried with filter paper, weighed, and immediately frozen and preserved at $-20^{\circ} \mathrm{C}$ until further analysis. Serum samples were collected after the centrifugation (Avanti J-E centrifuge, Beckman Coulter, Inc., USA) of the blood for $10 \mathrm{~min}$ at 3,000 rpm and stored at $-20^{\circ} \mathrm{C}$ for further analysis.

\section{Measurement of serum insulin level}

Serum insulin level was measured by an enzyme-linked immunosorbent assay (ELISA) procedure using mouse in- sulin (INS) ELISA kit (Linco Research Inc., Billerica, MA, USA).

\section{Determination of hepatic glycogen content}

Fresh liver was rinsed in $0.85 \%$ saline, while excess water was wiped by filter paper. Liver $(50 \mathrm{mg}$ ) was mixed with 3 time volume of $30 \% \mathrm{KOH}$ solution and heated by boiling water at $100^{\circ} \mathrm{C}$ for $20 \mathrm{~min}$, until the liver completely dissolved. The hepatic glycogen content was measured with a glycogen kit (Nanjing Jiancheng Bioengineering Institute, Nanjing, China) following the manufacturer's instruction.

\section{Lipid profile measurements}

Normal and experimentally induced type 2 diabetic mouse serum samples were analyzed to determine lipid profile levels. Total cholesterol (TC), triacylglycerol (TG), high-density lipoprotein (HDL) and low-density lipoprotein (LDL) were measured with commercial kits (Rongsheng Biotech, Co., Ltd., Shanghai, China) using an Automatic Biochemistry Analyzer (Hitachi 7020, Tokyo, Japan). Serum apolipoprotein A (apoA) and apolipoprotein B (apoB) levels were analyzed by the immunoturbidimetric immunoassay using a commercial kit (Nanjing Jiancheng Bioengineering Institute, Nanjing, China).

\section{Histological study}

After animals had been sacrificed, the livers from all mice were collected for histological analysis. The liver sections were fixed in $4 \%$ paraformaldehyde overnight and then embedded in paraffin. Those samples were stained with hematoxylin and eosin according to the routine protocol and examined under a light microscope (Humanoid Nikon E100, Tokyo, Japan).

\section{Statistical analysis}

All experiments were performed in duplicate. The data were analyzed by a statistical software package SPSS 19.0 for Windows. One-way ANOVA analysis was used to determine the significant difference between groups and Duncan's multiple range tests to perform multiple comparisons between means, and differences were considered to be statistical significant at $p<0.05$.

\section{RESULTS}

\section{Measurements of food intake, water consumption and body weight}

The main symptoms of type 2 diabetes include increased hunger, thirst and unexplained weight loss. Figure 1 shows the effects of feeding okara and soybean bran on food intake, water consumption and body weight of ICR mice with experimentally-induced type 2 diabetes. There was no significant difference in food intake between $\mathrm{HF}-\mathrm{OKG}$ and $\mathrm{HF}-\mathrm{MEG}$ per mouse per day over the 8-week experimental period, while the food intake was lower in HF-SBG, NCG and DCG than that in HF-OKG and HF-MEG (Figure 1A). The mice might not prefer the high-fat diet with soybean bran, compared to these with okara and metformin. The addition of okara into the high-fat diet significantly increased food intake by mice. 

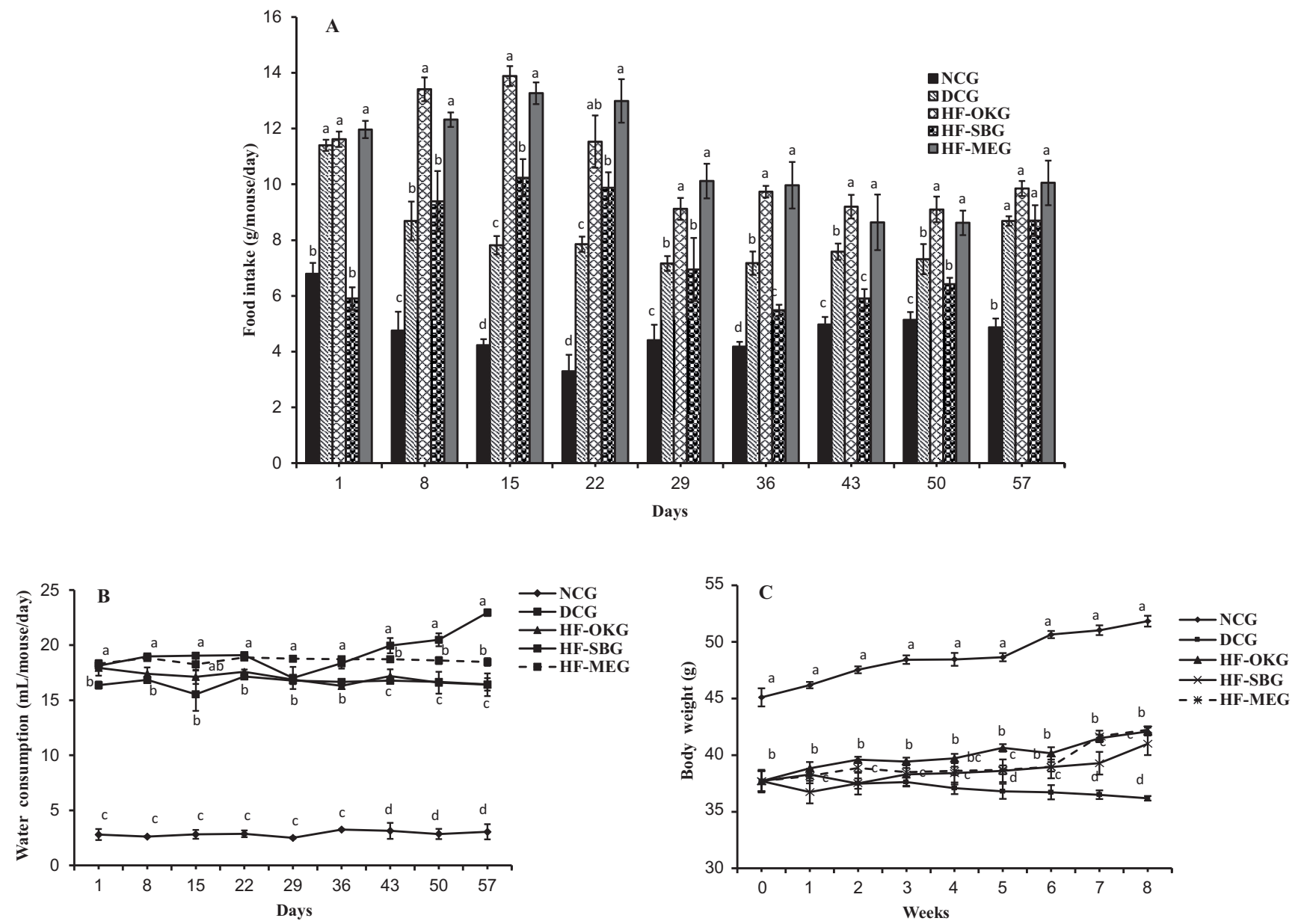

FIGURE 1. Effect of okara and soybean bran supplementation on general health condition of the experimentally-induced type 2 diabetes ICR mice. (A) Food intake. (B) Water consumption. (C) Weight gains. Different letters indicate significant differences $(p<0.05)$.

Figure 1B shows water consumption per mouse per day among different groups. The fluid intake was significantly higher in HF-OKG, HF-SBG, HF-MEG and DCG compared to NCG $(p<0.05)$ throughout the same period. Furthermore, there was a significant increase in water consumption by day 43 in DCG. There was no significant difference in water consumption between HF-OKG and HF-SBG, but the average amount of water consumption was significantly lower in HF-OKG and HF-SBG than DCG. The addition of okara to the diet caused health recovery in the mice, which resulted in lowering the water intake.

The normal control mice continued to increase weight consistently to the end of the experiment (Figure 1C). There was a significant difference in the body weight $(p<0.05)$ between the NCG and all diabetic groups during 8-week experimental period. HF-OKG, HF-SBG and HF-MEG gradually increased weight until the end of the experiment, while the diabetic control mice continued losing weight. Body weights of HF-OKG were significantly higher than those of HF-SBG. There was no significant difference in body weight between HF-SBG and HF-MEG during most of study period, while there was a significant difference between HF-MEG and HF-OKG in week 3 and 5 only. Okara, soybean bran or metformin gradually increased body weights of diabetic mice.

\section{Fasting blood glucose, oral glucose and insulin tolerance tests}

Measurement of fasting blood glucose is a simple analysis for detecting changes in glucose metabolism and is considered as the principal routine test for diabetes mellitus. Figure $2 \mathrm{~A}$ shows the effect of okara or soybean bran on blood glucose levels in ICR mice with experimentally-induced type 2 diabetes. The FBG levels in NCG were significantly lower than in all diabetic groups $(p<0.05)$. As expected, the FBG levels in the HF-OKG, HF-SBG and HF-MEG were decreased during 8-week period. After 3-week feeding with the diets containing okara, soybean bran or metformin, a significant reduction in FBG levels of mice was detected compared with the DCG. The impact of okara on FBG was similar to that of metformin but higher than of soybean bran.

The OGTT evaluates the disposal of a glucose load administered via oral dosing. Figure 2B illustrates the blood glucose responses to an OGTT after 7-week feeding experimental diets. The blood glucose level in DCG after a glucose load was higher than in all other groups. The blood glucose of DCG reached a peak value $30 \mathrm{~min}$ after glucose load and decreased to the lowest level at $120 \mathrm{~min}$. The HF-OKG, HF-SBG and HF-MEG showed a significant increase in the rate of glucose clearance as compared to DCG. Okara, soybean bran 
and metformin showed the similar glucose clearance rate, but a significant difference in blood glucose level appeared between NCG and those diet groups. Okara, soybean bran and metformin result in a good disposal of a glucose load compared to DCG that were kept on a high-fat diet.

The ITT monitors glucose concentration over time, in response to insulin. Figure $2 \mathrm{C}$ shows the results of the insulin response test among different groups. The insulin tolerance test was conducted on 4-h fasted mice. Initially, the blood glucose concentration was the lowest in NCG and the highest in DCG. In the diabetic control group, the blood glucose concentrations were increased after injecting insulin and extended until $60 \mathrm{~min}$, then slightly decreased at $120 \mathrm{~min}$. The blood glucose levels in HF-OKG, HF-SBG and HF-MEG decreased successively after injecting insulin, indicating that they had higher sensitivity of response to the insulin than DCG mice.
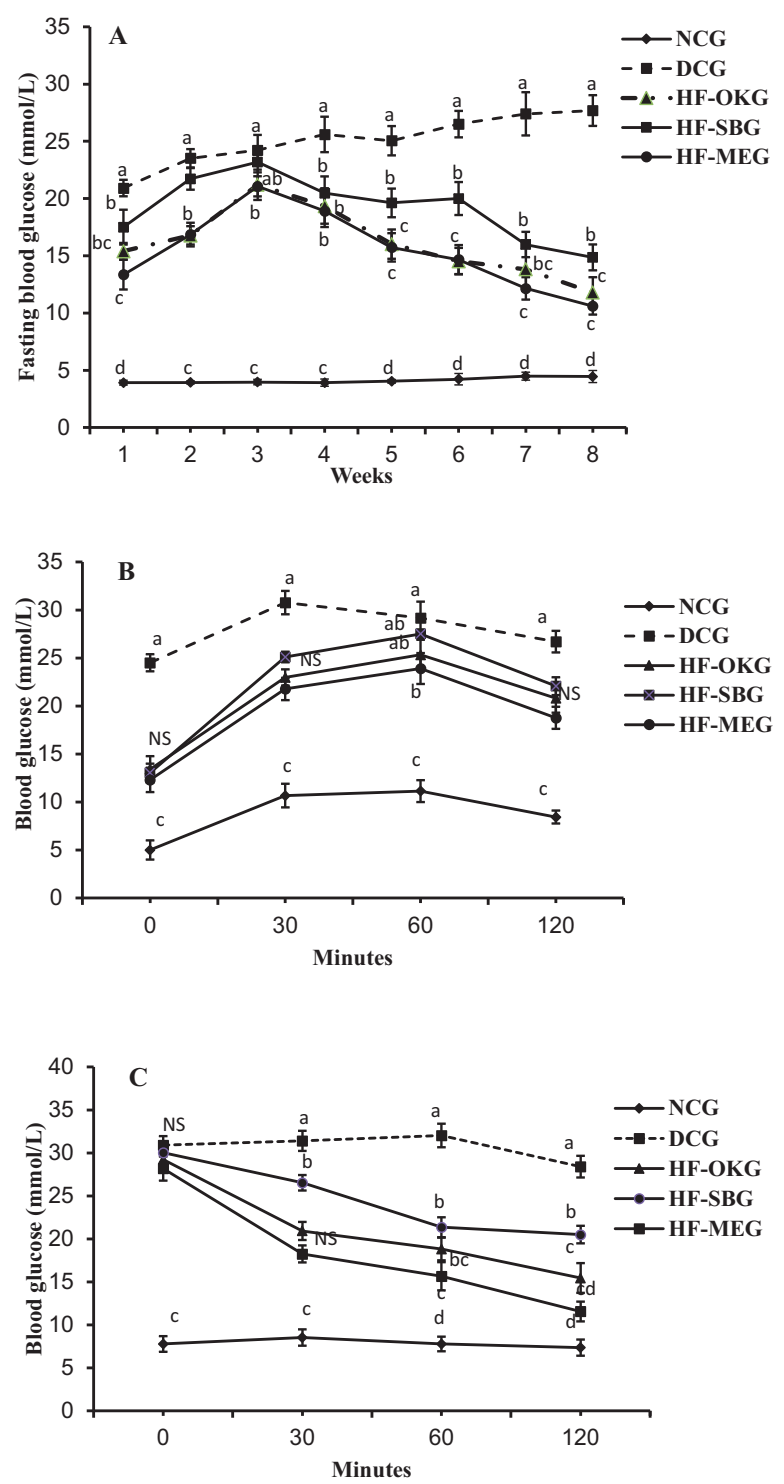

FIGURE 2. Blood glucose and insulin responses in experimentally-induced type 2 diabetes ICR mice with different treatments over the 8 weeks experimental period. (A) The fasting blood glucose measurement. (B) The oral glucose tolerance test. (C) The insulin tolerance test. Different letters indicate significant differences $(p<0.05)$.
In the HF-SBG, however, the decrease of the blood glucose showed a delay compared with $\mathrm{HF}-\mathrm{OKG}$ and HF-MEG. The response of $\mathrm{HF}-\mathrm{OKG}$ to the administered insulin was more sensitive than that of HF-SBG.

\section{Measurements of serum insulin and hepatic glycogen}

Serum insulin level is detected for the glucose metabolism changes in the diabetic subject. Figure 3A shows the effect of okara or soybean bran supplementation on serum insulin levels in experimentally-induced type 2 diabetic ICR mice. Serum insulin levels were significantly lower in DCG as compared with $\mathrm{NCG}, \mathrm{HF}-\mathrm{OKG}, \mathrm{HF}-\mathrm{SBG}$ and $\mathrm{HF}-\mathrm{MEG}$ after 8-week feeding with test diets, while serum insulin level of HF-OKG, HF-SBG or HF-MEG was significantly lower than that of NCG. Okara and soybean bran had a similar effect on insulin level, but both were less effective than metformin. Therefore, okara and soybean bran could enhance the insulin secretion.

Glycogen content can be significantly changed upon glucose homeostasis disruption in diabetes. Figure $3 \mathrm{~B}$ shows the effect of okara, soybean bran and metformin on hepatic glycogen content. Liver glycogen content was significantly higher in HF-OKG, HF-SBG, HF-MEG or NCG than the one in DCG. However, glycogen content in HF-SBG was similar to that in NCG but higher than that in $\mathrm{HF}-\mathrm{OKG}$ or HF-MEG. Results indicated that soybean bran and okara positively regulated the glycogenesis pathway in the liver through improving the insulin secretion.
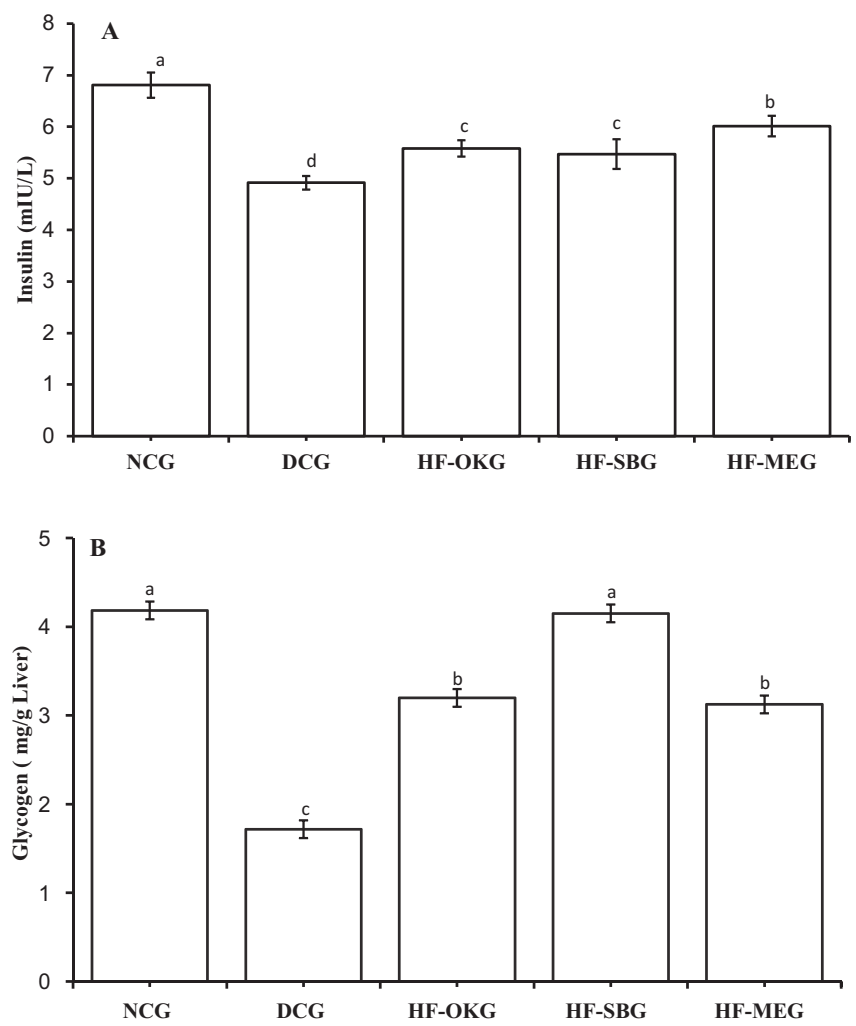

FIGURE 3. The effects of feeding okara or soybean bran on serum insulin and hepatic glycogen measurements. (A) Serum insulin levels. (B) Hepatic glycogen contents. Different letters indicate significant differences $(p<0.05)$ 

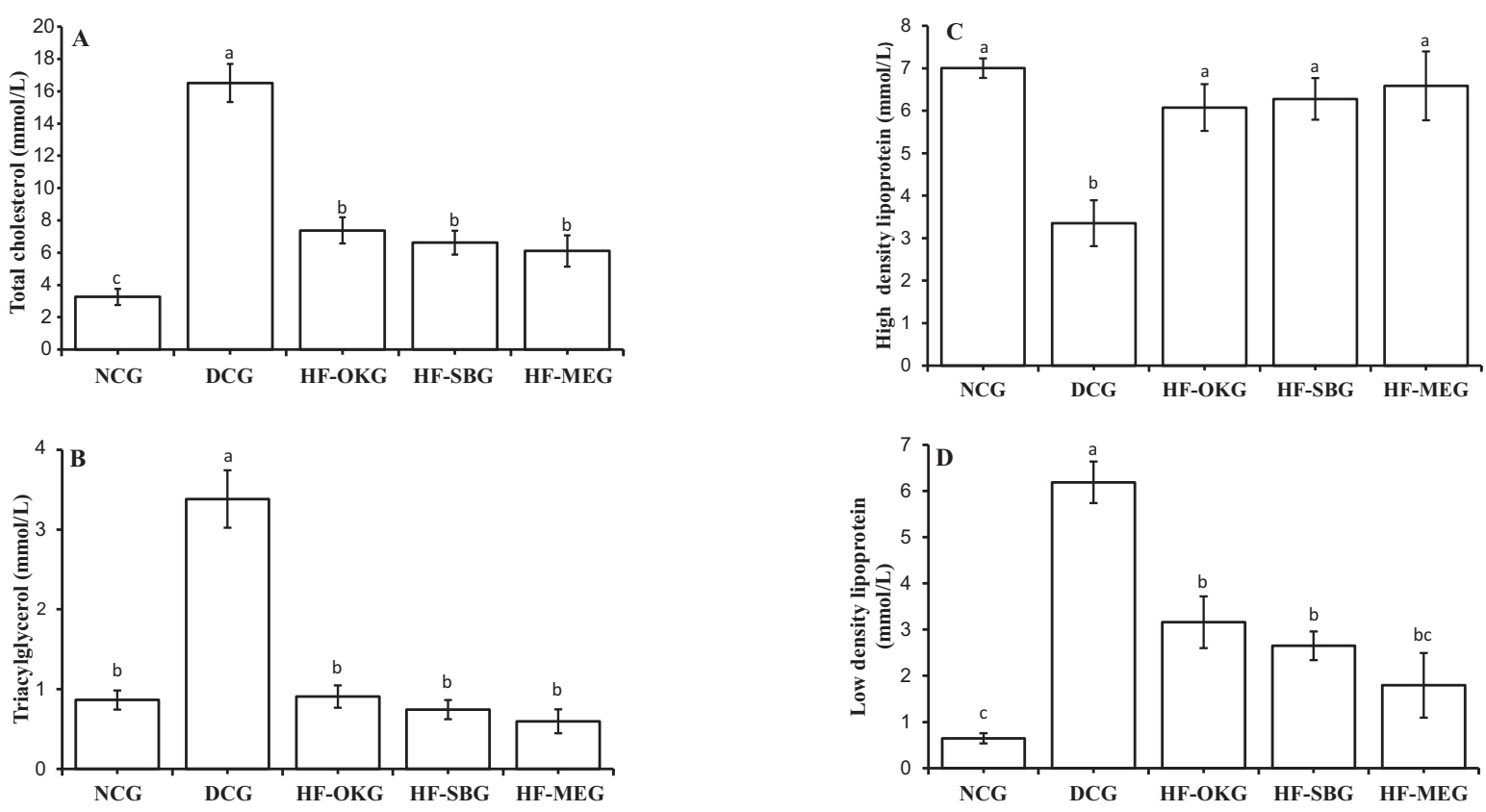

FIGURE 4. Effect of okara and soybean bran supplementation on lipid profiles measurement in experimentally-induced type 2 diabetes ICR mice. (A) Total cholesterol. (B) Triacylglycerol. (C) High density lipoprotein. (D) Low density lipoprotein. Different letters indicate significant differences $(p<0.05)$.

\section{Lipid profile measurements}

Comparing to normal, the diabetic mice usually have lower high-density lipoprotein and higher total cholesterol, triacylglycerol, and low-density lipoprotein and this is mainly a consequence of insulin resistance, which affects enzyme and lipid metabolism [Bardini et al., 2012]. Figure 4 shows the lipid profiles of the five groups at the end of the experimental period. TC (Figure 4A), TG (Figure 4B) and LDL (Figure 4D) levels were significantly lower in HF-OKG or HF-SBG than in DCG, but higher than in NCG. The HF-OKG or HF-SBG had a higher HDL level than DCG (Figure 4C), but lower than that in NCG and HF-MEG.

In general, the apoA level is significantly reduced in diabetics, but the improvement of glycemic control raises the apoA levels. Table 2 demonstrates the effect of okara and soybean bran on apo lipoprotein $\mathrm{A}$ and apo lipoprotein B secretion in mice with experimentally-induced type 2 diabetes. In this study, apoA concentration was significantly increased in mice fed the normal diet, HFD supplemented with okara, soybean bran or metformin, compared with the DCG fed HFD only. Conversely, a significant difference was observed in the final values of the apoB concentration among all diabetic groups (Table 2). The apoB/apoA ratio values of all mice in different groups indicated that feeding the normal diet, HFD supplemented with okara, soybean bran or metformin caused a significant decrease in apoB/ apoA ratio compared with the diabetic mice fed HFD only (Table 2).

\section{Organs and epididymal fat weights}

Table 3 illustrates the relative weight comparisons of liver, epididymal fat, heart, kidney, pancreas and spleen of mice with different treatments. The relative liver weight of mice in DCG was significantly higher than in all other groups $(p<0.05)$. Okara and soybean bran had similar effects on relative liver weight. There was a significant difference in the relative epididymal fat weight between NCG and other diabetic groups. The relative heart weight in DCG was significantly lower than in other groups. There was a significant difference $(p<0.05)$ in NCG, HF-OKG and HF-SBG relative kidney weight compared to DCG and HF-MEG. The pancreas relative weight was significantly different in NCG, HF-OKG, HF-SBG and HF-MEG compared to DCG. There was no significant difference in relative spleen weight

TABLE 2. Comparison of serum apolipoprotein A (apo A), apolipoprotein B (apo B) and the ratio of apo B/apo A in normal, diabetic and treated diabetic mice.

\begin{tabular}{l|c|c|c|c|c}
\hline Lipid parameters & NCG & DCG & HF-OKG & HF-SBG & HF-MEG \\
\hline apo A $(\mathrm{g} / \mathrm{L})$ & $0.19 \pm 0.02^{\mathrm{a}}$ & $0.04 \pm 0.0003^{\mathrm{d}}$ & $0.13 \pm 0.02^{\mathrm{b}}$ & $0.07 \pm 0.0003^{\mathrm{c}}$ & $0.14 \pm 0.02^{\mathrm{b}}$ \\
apo B $(\mathrm{g} / \mathrm{L})$ & $0.07 \pm 0.002^{\mathrm{d}}$ & $0.12 \pm 0.001^{\mathrm{a}}$ & $0.11 \pm 0.0003^{\mathrm{b}}$ & $0.06 \pm 0.001^{\mathrm{d}}$ & $0.10 \pm 0.01^{\mathrm{c}}$ \\
apo B/apo A ratio & $0.35 \pm 0.18^{\mathrm{b}}$ & $3.10 \pm 0.14^{\mathrm{a}}$ & $0.95 \pm 0.69^{\mathrm{b}}$ & $0.99 \pm 0.24^{\mathrm{b}}$ & $0.78 \pm 0.25^{\mathrm{b}}$ \\
\hline
\end{tabular}

Values (row) not sharing a common alphabet are significantly different at $p<0.05$. 
TABLE 3. Relative organs and epididymal fat weight in normal, diabetic and treated diabetic mice ${ }^{\mathrm{a}}$.

\begin{tabular}{|c|c|c|c|c|c|c|}
\hline Group & Liver & Epididymal fat & Heart & Kidney & Pancreas & Spleen \\
\hline $\mathrm{NCG}$ & $3.40 \pm 0.1^{\mathrm{d}^{*}}$ & $3.24 \pm 0.46^{\mathrm{a}}$ & $0.44 \pm 0.09^{\mathrm{a}}$ & $1.56 \pm 0.06^{\mathrm{b}}$ & $0.26 \pm 0.012^{\mathrm{c}}$ & $0.20 \pm 0.012^{\mathrm{d}}$ \\
\hline $\mathrm{DCG}$ & $12.40 \pm 0.6^{\mathrm{a}}$ & $0.70 \pm 0.07^{\mathrm{b}}$ & $0.38 \pm 0.03^{\mathrm{b}}$ & $1.84 \pm 0.09^{\mathrm{a}}$ & $0.35 \pm 0.01^{\mathrm{a}}$ & $0.34 \pm 0.022^{\mathrm{a}}$ \\
\hline $\mathrm{HF}-\mathrm{OKG}$ & $7.50 \pm 0.4^{\mathrm{bc}}$ & $0.68 \pm 0.63^{\mathrm{b}}$ & $0.50 \pm 0.01^{\mathrm{a}}$ & $1.64 \pm 0.05^{\mathrm{b}}$ & $0.29 \pm 0.02^{\mathrm{b}}$ & $0.30 \pm 0.007^{\mathrm{b}}$ \\
\hline HF-SBG & $7.74 \pm 0.7^{b}$ & $0.34 \pm 0.09^{b}$ & $0.44 \pm 0.02^{\mathrm{a}}$ & $1.54 \pm 0.08^{\mathrm{b}}$ & $0.30 \pm 0.03^{\mathrm{b}}$ & $0.30 \pm 0.012^{b}$ \\
\hline HF-MEG & $6.96 \pm 0.6^{c}$ & $0.56 \pm 0.13^{\mathrm{b}}$ & $0.50 \pm 0.09^{\mathrm{a}}$ & $1.28 \pm 0.23^{\mathrm{c}}$ & $0.28 \pm 0.02^{\mathrm{bc}}$ & $0.24 \pm 0.013^{\mathrm{c}}$ \\
\hline
\end{tabular}

aRelative organ weight $(\%)=$ Organ weight in gram/body weight in gram $\times 100$. "Values (column) not sharing a common alphabet within the same column are significantly different at $p<0.05$.

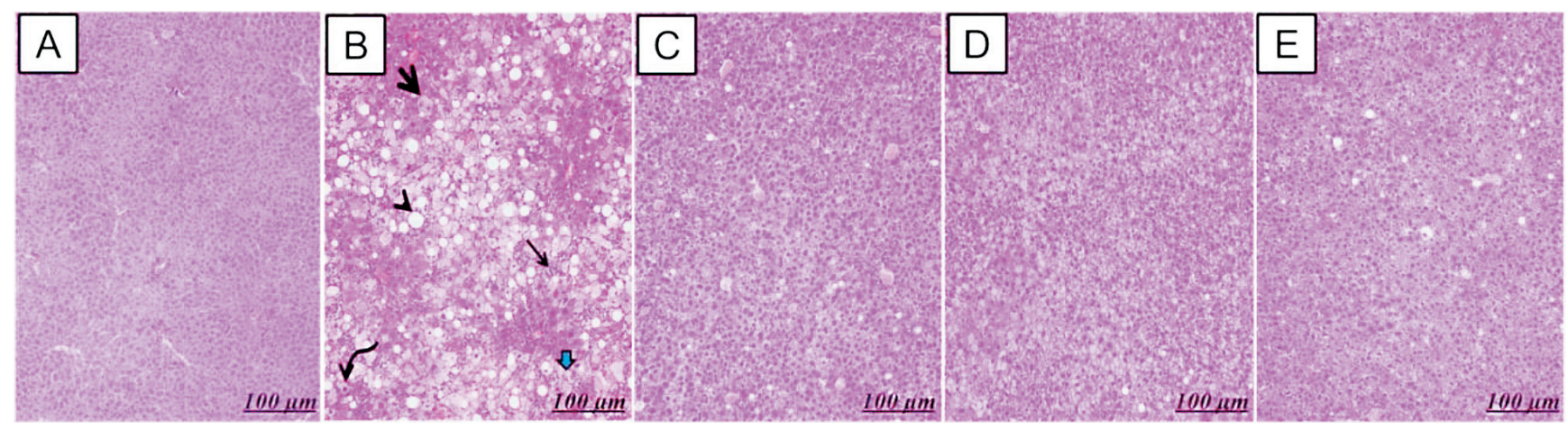

FIGURE 5. Histopathological sections of livers. (A) NCG. (B) DCG. (C) HF-MEG. (D) HF-SBG. (E) HF-OKG. Different signs indicated severe degeneration (curved arrow), lipid droplets (arrow head), necrosis (right arrow), hepatocellular swelling (short arrow) and inflammatory cell infiltrate (long arrow).

among HF-OKG and HF-SBG. However, the relative weight of spleen was significantly higher in DCG compared to other diabetic groups and NCG. Liver and kidney relative weights were higher in STZ-induced diabetic animals but the feeding of okara or soybean bran supplementation reversed the increase in the relative weights of these organs.

\section{Histological study}

Figure 5 demonstrates the histological changes in hepatocytes of the five groups at the end of the experimental period. Examination of mouse liver biopsy revealed normal arrangement of the hepatocytes with no visible lesion in liver of NCG (Figure 5A), while hepatocytes in the DCG showed severe degeneration, numbers of lipid droplets, necrosis, hepatocellular swelling and inflammatory cell infiltrate (Figure 5B). Liver cells from HF-MEG (Figure 5C) were arranged neatly and reduced or eliminated lipid droplets without significant inflammation or hepatocellular swelling. A similar improvement was observed among the HF-SBG (Figure 5D) and the HF-OKG (Figure 5E). Interestingly, administration of soybean bran or okara effectively decreased lipid droplets without significant inflammation or hepatocellular swelling. However, soybean bran was more effective than okara in reducing hepatic cell histological changes. The histological examination of mouse hepatic tissues in HF-SBG showed less lipid droplets compared to HF-OKG. Therefore, effects of okara and soybean bran on liver suggested their favorable hepatoprotective effects on mice with type 2 diabetes induced by a high-fat diet and streptozotocin.

\section{DISCUSSION}

In this study, normal or experimentally-induced type 2 diabetes ICR male mice were fed normal or HFD supplemented with high fiber by-products (soybean bran or okara) for eight weeks, to validate the hypothesis that high-fiber diet would have hypoglycemic effects on diabetic mice, and to provide experimental evidence for developing suitable foods for type 2 diabetes mellitus patients from the high-fiber diet.

It is well known that polyuria, polydipsia, polyphagia and weight loss represent the initial signs of diabetes in humans as well as in animal models [Kavey et al., 2006]. The quantities of food and water intake of diabetic mice were much higher than these of the normal mice [Lim \& Lee, 2010]. The present results demonstrated that there was a significant increase of food intake in HF-OKG compared to HF-SBG during the whole experimental period. It indicated that the addition of okara could increase the diet's palatability. Thirst in diabetic status is mainly caused by degenerative changes in metabolism [Lim \& Lee, 2010]. In this study, the amount of water consumption was significantly lower in HF-OKG and HF-SBG than DCG. It indicated that the increase of okara and soybean bran in the diet would impact a decrease in water consumption.

STZ-induced diabetes is associated with a severe reduction in body weight, due to the degradation or loss of structural proteins that are obviously known to contribute to the body weight [Li et al., 2011]. During this study, DCG continued to lose weight and NCG continued to gain weight and this agreed with results of Ahmed et al. [2010], who found a sig- 
nificant difference between normal control mice and diabetic control mice body weight. A gradual increase in body weight was observed in HF-OKG, HF-SBG and HF-MEG due to an improvement in diabetes control and alleviating of diabetes-associated weight loss. The HF-SBG showed considerably lower body weight than the $\mathrm{HF}-\mathrm{OKG}$, suggesting that soybean bran could control the increase in body weight of mice under HFD condition. Finger millet bran supplementation prevented body weight gain in HFD-fed mice [Murtaza et al., 2014]. The mechanisms, by which fiber sources lower body weight, are associated with raising satiety, reducing hunger, providing a feeling of fullness and playing a role in the control of energy balance [Schroeder et al., 2013].

Feeding okara and soybean bran resulted in a remarkable fall in FBG level in the experimentally-induced type 2 diabetes ICR mice, compared to diabetic control mice. This effect was higher in $\mathrm{HF}-\mathrm{OKG}$ than HF-SBG. The beneficial effect of okara on reducing the blood glucose level was due to its content of dietary fiber [Lu et al., 2013] and also its protein content [Ahmad et al., 2010]. The postprandial serum glucose level of mice fed with soybean bran was distinctly slower than others fed the diet without fiber, probably due to its high amount of dietary fiber [Mahalko et al., 1984]. The soybean dietary fiber contains bioactive components with high viscosity, while these components could delay gastric emptying and glucose absorption [Chang et al., 2008]. Therefore, the hypoglycemic effect of okara and soybean bran dietary fiber could be due to reduced glucose absorption by slowing gastric emptying. The variation of okara and soybean bran effects on FBG might be due to that okara contains more protein than soybean bran and produces some effect on blood glucose levels of mice fed diet containing it.

Impaired glucose tolerance is one of the main characteristics of insulin resistance [Chen et al., 2014]. Soy protein can improve glucose tolerance [Ahmad et al., 2014]. OGTT results showed that okara delayed the increase of postprandial glucose level and significantly reduced postprandial glucose peak versus DCG. However the peak was still considerably higher than that of NCG. The mice fed soybean bran had a higher blood glucose level compared to okara-fed mice. Feeding short chain fructo-oligosaccharides for 4 to 6 weeks improved glucose tolerance in STZ-treated diabetic rats [Cani et al., 2005].

Consumption of rich oat or barley beta-glucan foods improved insulin response in type 2 diabetes subjects [Zheng et al., 2013]. The same effect was further confirmed in type 2 diabetic mice model by feeding the resistant maltodextrin [He et al., 2015]. In this study, the immediate decrease of blood glucose concentrations in $\mathrm{HF}-\mathrm{OKG}, \mathrm{HF}-\mathrm{SBG}$ and HF-MEG implied the improvement of their sensitive responses to the administered insulin compared to DCG. Moreover, the decrease of the blood glucose effect caused by soybean bran showed a distinct delay compared with okara.

In this study, the supplementation of all treatments increased the serum insulin level of the STZ-induced diabetic mice compared to those fed with HFD alone. Similar improvements occurred also in diabetic rats and mice fed with fenugreek [El Rahman, 2014] and fermented soybean extract [Nam et al., 2012], which caused an increase in the produc- tion of insulin by keeping pancreatic beta cells and encouraging their glucose-dependent insulin secretion. Therefore, the antihyperglycemic activities of okara and soybean bran were associated with an increase in serum insulin level, while okara and soybean bran might probably cause an improvement in insulin sensitivity and subsequent reduction of hyperglycemia and glucotoxicity.

Compared to the normal, the glycogen content of the liver is reduced in individuals with type 2 diabetes [Klover \& Mooney, 2004]. In this study, the hepatic glycogen increased significantly in experimentally-induced type 2 diabetes mice fed okara or soybean bran. This might be caused by reactivation of glycogen synthase duo to the increase of insulin secretion. Soluble dietary fiber fraction from fenugreek administration also increased glycogen content in type 2 diabetic rats [Hannan et al., 2007], while supplementation with soybean fermented paste, rice bran or phytic acid resulted in a higher content of hepatic glycogen than that of high-fat-fed mice [Chung et al., 2014; Kim et al., 2010].

Compared to normal, the diabetic mice usually have lower HDL and higher TC, TG, and LDL and this is mainly a consequence of insulin resistance, which affects enzyme and lipid metabolisms [Bardini et al., 2012]. One of the main health benefits of dietary fiber is related to its role as a hypolipidemic agent. Studies investigating the hypolipidemic effect of dietary fiber showed a potential decrease of lipid anomalies in diabetic mice fed high-fiber diet [Ismaiel et al., 2016]. Results of the present study demonstrate that feeding okara and soybean bran had a potential hypolipidemic effect by causing significant reduction of serum TC, TG and LDL levels while increasing HDL level to values similar to those in NCG. However, the hypolipidemic effects of okara and soybean bran were less immediate and intensive than that of metformin-treated mice. Okara supplementation increased the messenger RNA (mRNA) expressions of genes related to hepatic $\beta$-oxidation resulting in lower serum TC, TG, and LDL in mice [Kim et al., 2016]. Thus okara might regulate lipid metabolism by increasing the fat excretion and decreasing serum lipids.

Cardiovascular disease is more prevalent among patients with type 2 diabetes, and apoB/apoA ratio is the accurate measurable factor to predict cardiovascular risk [Dahlén et al., 2009]. In this study, the apoB/apoA ratio values of all mice in different groups indicated that feeding HFD supplemented with okara, soybean bran or metformin caused a significant decrease in apoB/apoA ratio compared with the diabetic mice fed HFD only. The hypolipidemic effect of okara does not seem to be due only to one component, but rather to the complement action of its dietary fiber and protein. The main components of okara, dietary fiber and protein could be related to the total lipids and cholesterol decrease in the plasma [Villanueva et al., 2011]. Previous clinical study targeting the effect of soybean bran on lipid profile indicated that soy protein intake could decrease intestinal cholesterol absorption, enhance bile acid secretion, increase removal of LDL and reduce hepatic cholesterol content [Ajay et al., 2011].

Alteration of the internal organ weights indicated occurrence of either pathology or toxicity to these organs. Type 2 diabetes is a chronic disease that affects multiple organ systems and is associated with the generation of reactive oxygen 
species [Kaneto et al., 2010], while it can cause oxidative damage, particularly to liver, kidney and heart. The accumulation of liver fat led to an increase in liver weight [Lim \& Lee, 2010]. Insulin resistance increased the hepatic production of TG-rich particles in liver [Lee et al., 2008]. In this study, okara and soybean bran significantly reduced relative liver weight compared to the DCG, which may be attributed to the decrease of TG levels in the HF-OKG and HF-SBG. Furthermore, diabetes can cause the polyuria, which affects the kidney function, increases the stress and enlarges the kidney [Lim \& Lee, 2010]. Therefore, the weight of kidney with DCG was the highest, because the increase of kidney weight in diabetes was correlated to the increase of protein and lipid synthesis [Moodley et al., 2015]. The reduced kidney relative weight in the HF-OKG and HF-SBG showed the potential improvements of okara and soybean bran on the kidney. Therefore, these effects on those organs were considered a direct evidence of improved diabetic hepatopathies and nephropathies.

Currently, T2DM has become one of the most important causes of liver disease, such as nonalcoholic fatty liver disease, abnormal liver enzymes, hepatocellular carcinoma, cirrhosis and acute liver failure [Tolman et al., 2007]. The degenerative changes in the histology of liver of STZ-induced type 2 diabetic ICR mice were similar to previous observations [Teoh et al., 2008]. Histologically, liver section of STZ-induced diabetic mice showed severe degeneration, necrosis, hepatocellular swelling, inflammatory cell infiltrate and a lot of large lipid droplets in the cytoplasm and those symptoms were strongly correlated with insulin resistance [Han et al., 2015]. This damage was partially reversed by feeding okara and soybean bran and was similar to an observation that soybean treatment decreased the pathogenic effect and removed the fatty change in liver of diabetic rats [Amer, 2012]. This result indicated that okara and soybean bran might have the cytoprotective effect. However, soybean bran was more effective than okara in alleviating hepatic cell histological changes.

Metformin is considered the drug of choice for type 2 diabetes treatment, acting by enhancing glucose uptake and insulin sensitivity as well as decreasing rates of glucose production through a reduction of intracellular glucose production [Correia et al., 2008]. A recent research demonstrated that the okara treatment significantly increased the mRNA expression levels of peroxisome proliferator-activated receptors $\gamma$ (PPAR $\gamma)$, adiponectin and glucose transporter type 4 in the white adipose tissues of Goto-Kakizaki rats and suggested that the okara hypoglycemic effects might be through the PPAR $\gamma$-mediated pathway [Hosokawa et al., 2016]. Based on the current study results, okara and soybean bran hypoglycemic action might be associated with different mechanisms, such as reducing glucose absorption from intestine, increasing glycogenesis and lipid metabolism, enhancing pancreatic secretion of insulin and recovery of partially destroyed beta cells and increasing peripheral insulin sensitivity.

\section{CONCLUSIONS}

In conclusion, okara or soybean bran at a dose of $15 \%$ could significantly reduce the blood glucose level, prevent the increase of serum cholesterol, triacylglycerol and low density lipoprotein, reduce liver and kidney weights, and improve hepatic pathological symptoms in experimentally-induced type 2 diabetes ICR mice. Results indicated that okara and soybean bran could potentially offer the beneficial features for the T2DM patients.

\section{RESEARCH FUNDING}

The research was carried out in the framework of the research task financed by the Fundamental Research Funds for the Central Universities (Project 2013PY096).

\section{CONFLICT OF INTEREST}

Authors declare no conflict of interest.

\section{REFERENCES}

1. Ahmad A., Hayat I., Arif S., Masud T., Khalid N., Ahmed A., Mechanisms involved in the therapeutic effects of soybean (Glycine Max). Int. J. Food Prop., 2014, 17, 1332-1354.

2. Ahmad L., Hassan D., Hemeda H., Antihyperglycemic effects of okara, corn hull and their combination in alloxan induced diabetic rats. World Appl. Sci. J., 2010, 9, 10, 1139-1147.

3. Ajay K., Athiny X., Navin K., Rakesh K., Soybean constituents and their functional benefits. 2011, in: Opportunity, Challenges and Scope of Natural Products in Medicinal Chemistry (ed. V.K. Tiwari). Research Signpost, Kerala, India, pp. 367-383.

4. Amer N., Effects of soybean seed on glucose levels, lipid profiles and histological structures of the liver in alloxan-induced diabetic albino rats. Tikrit J. Pure Sci., 2012, 17, 1-5.

5. Anderson J.W., Ward K., High-carbohydrate, high-fiber diets for insulin-treated men with diabetes mellitus. Am. J. Clin. Nutr., 1979, 32, 2312-2321.

6. Bardini G., Rotella C.M., Giannini S., Dyslipidemia and diabetes: reciprocal impact of impaired lipid metabolism and Beta-cell dysfunction on micro-and macrovascular complications. Rev. Diabet. Stud., 2012, 9, 82-93.

7. Cani P.D., Daubioul C.A., Reusens B., Remacle C., Catillon G., Delzenne N.M., Involvement of endogenous glucagon-like peptide-1 (7-36) amide on glycaemia-lowering effect of oligofructose in streptozotocin-treated rats. J. Endocrinol., 2005, 185, 457-465.

8. Cannon M., Flenniken A., Track N.S., Demonstration of acute and chronic effects of dietary fibre upon carbohydrate metabolism. Life Sci., 1980, 27, 1397-1401.

9. Chang J.H., Kim M.S., Kim T.W., Lee S.S., Effects of soybean supplementation on blood glucose, plasma lipid levels, and erythrocyte antioxidant enzyme activity in type 2 diabetes mellitus patients. Nutr. Res. Pract., 2008, 2, 152-157.

10. Chen P., Zhang Q., Dang H., Liu X., Tian F., Zhao J., Chen Y., Zhang H., Chen W., Antidiabetic effect of Lactobacillus casei CCFM0412 in high-fat-fed, streptozotocin-induced type 2 diabetic mice. Nutrition, 2014, 30, 1061-1068.

11. Chung S.I., Rico C.W., Kang M.Y., Comparative study on the hypoglycemic and antioxidative effects of fermented paste (doenjang) prepared from soybean and brown rice mixed with rice bran or red ginseng marc in mice fed with high-fat diet. Nutrients, 2014, 6, 4610-4624. 
12. Cicek B., Arslan P., Fahretti K., The effects of oligofrutose and polydextrose on metabolic control parameters in type-2 diabetes. Pak. J. Med. Sci., 2009, 25, 573-578.

13. Correia S., Carvalho C., Santos M.S., Seica R., Oliveira C.R., Moreira P.I., Mechanisms of action of metformin in type 2 diabetes and associated complications: an overview. Mini. Rev. Med. Chem., 2008, 8, 1343-1354.

14. Dahlén E.M., Länne T., Engvall J., Lindström T., Grodzinsky E., Nystrom F., Östgren C.J., Carotid intima-media thickness and apolipoprotein B/apolipoprotein A-I ratio in middle-aged patients with type 2 diabetes. Diabet. Med., 2009, 26, 384-390.

15. Dueñas M., Hernández T., Robredo S., Lamparski G., Estrella I., Muñoz R., Bioactive phenolic compounds of soybean (Glycine max cv. Merit): modifications by different microbiological fermentations. Pol. J. Food Nutr. Sci., 2012, 62, 241-250.

16. El Rahman A.M.A., Hypoglycemic and hypolipidemic effect of fenugreek in different forms on experimental rats. World Appl. Sci. J., 2014, 29, 7, 835-841.

17. Han S., Jiao, J., Zhang W., Xu J., Wan Z., Zhang W., Gao X., and Qin L., Dietary fiber prevents obesity-related liver lipotoxicity by modulating sterol-regulatory element binding protein pathway in C57BL/6J mice fed a high-fat/cholesterol diet. Sci. Rep., 2015, 5, 15256.

18. Hannan J.M., Ali L., Rokeya B., Khaleque J., Akhter M., Flatt P.R., Abdel-Wahab Y.H., Soluble dietary fibre fraction of Trigonella foenum-graecum (fenugreek) seed improves glucose homeostasis in animal models of type 1 and type 2 diabetes by delaying carbohydrate digestion and absorption, and enhancing insulin action. Br. J. Nutr., 2007, 97, 514-521.

19. He B., Nohara K., Ajami N.J., Michalek R.D., Tian X., Wong M., Losee-Olson S.H., Petrosino J.F., Yoo S.-H., Shimomura K., Transmissible microbial and metabolomic remodeling by soluble dietary fiber improves metabolic homeostasis. Sci. Rep., 2015, 5,10604 .

20. Hosokawa M., Katsukawa M., Tanaka H., Fukuda H., Okuno S., Tsuda K., Iritani N., Okara ameliorates glucose tolerance in GK rats. J. Clin. Biochem. Nutr., 2016, 58, 216-222.

21. Ismaiel M., Yang H., Min C., Dietary fiber role in type 2 diabetes prevention. Br. Food J., 2016, 118, 961-975.

22. Jin L., Tu J., Jia J., An W., Tan H., Cui Q., Li Z., Drug-repurposing identified the combination of trolox $\mathrm{C}$ and cytisine for the treatment of type 2 diabetes. J. Transl. Med., 2014, 12, 153.

23. Kaneto H., Katakami N., Matsuhisa M., Matsuoka T.-A., Role of reactive oxygen species in the progression of type 2 diabetes and atherosclerosis. Mediators Inflamm., 2010, 2010, 453892.

24. Kavey R.-E.W., Allada V., Daniels S.R., Hayman L.L., McCrindle B.W., Newburger J.W., Parekh R.S., Steinberger J., Cardiovascular risk reduction in high-risk pediatric patients: a scientific statement from the american heart association expert panel on population and prevention science; the councils on cardiovascular disease in the young, epidemiology and prevention, nutrition, physical activity and metabolism, high blood pressure research, cardiovascular nursing, and the kidney in heart disease; and the interdisciplinary working group on quality of care and outcomes research: endorsed by the American Academy of Pediatrics. Circulation, 2006, 114, 2710-2738.

25. Kiehm T.G., Anderson J.W., Ward K., Beneficial effects of a high carbohydrate, high fiber diet on hyperglycemic diabetic men. Am. J. Clin. Nutr., 1976, 29, 895-899.
26. Kim H.-S., Yu O.-K., Byun M.-S., Cha, Y.-S., Okara, a soybean by-product, prevents high-fat diet-induced obesity and improves serum lipid profiles in C57BL/6J mice. Food Sci. Biotechnol., 2016, 25, 607-613.

27. Kim S.M., Rico C.W., Lee S.C., Kang M.Y., Modulatory effect of rice bran and phytic acid on glucose metabolism in high-fat-fed C57BL/6N mice. J. Clin. Biochem. Nutr., 2010, 47, 12-17.

28. Klover P.J., Mooney R.A., Hepatocytes: critical for glucose homeostasis. Int. J. Biochem. Cell. Biol., 2004, 36, 753-758.

29. Lee Y.A., Cho E.J., Yokozawa T., Effects of proanthocyanidin preparations on hyperlipidemia and other biomarkers in mouse model of type 2 diabetes. J. Agric. Food Chem., 2008, 56, 7781-7789.

30. Lemes S.F., Lima F.M., de Almeida A.P.C., Ramalho A.d.F.S., de Lima Reis S.R., Michelotto L.F., Amaya-Farfán J., Carneiro E.M., Boschero A.C., Latorraca M.Q., Nutritional recovery with okara diet prevented hypercholesterolemia, hepatic steatosis and glucose intolerance. Int. J. Food Sci. Nutr., 2014, 65, 745-753.

31. Li F., Zhang Y., Zhong Z., Antihyperglycemic effect of Ganoderma lucidum polysaccharides on streptozotocin-induced diabetic mice. Int. J. Mol. Sci., 2011, 12, 6135-6145.

32. Lim S.-I., Lee B.-Y., Anti-diabetic effect of material fermented using rice bran and soybean as the main ingredient by Bacillus $s p$. J. Korean Soc. Appl. Biol. Chem., 2010, 53, 222-229.

33. Lu F., Liu Y., Li B., Okara dietary fiber and hypoglycemic effect of okara foods. Bioact. Carbohydr. Diet. Fibre, 2013, 2, 126-132.

34. Madar Z., Effect of brown rice and soybean dietary fiber on the control of glucose and lipid metabolism in diabetic rats. Am. J. Clin. Nutr., 1983, 38, 388-393.

35. Mahalko J.R., Sandstead H.H., Johnson L.K., Inman L.F., Milne D.B., Warner R.C., Haunz E.A., Effect of consuming fiber from corn bran, soy hulls, or apple powder on glucose tolerance and plasma lipids in type II diabetes. Am. J. Clin. Nutr., 1984, $39,25-34$.

36. Moodley K., Joseph K., Naidoo Y., Islam S., Mackraj I., Antioxidant, antidiabetic and hypolipidemic effects of Tulbaghia violacea harv. (wild garlic) rhizome methanolic extract in a diabetic rat model. BMC Complement Altern. Med., 2015, 15, 408.

37. Murotomi K., Umeno A., Yasunaga M., Shichiri M., Ishida N., Koike T., Matsuo T., Abe H., Yoshida Y., Nakajima Y., Oleuropein-rich diet attenuates hyperglycemia and impaired glucose tolerance in type 2 diabetes model mouse. J. Agric. Food Chem., 2015, 63, 6715-6722.

38. Murtaza N., Baboota R.K., Jagtap S., Singh D.P., Khare P., Sarma S.M., Podili K., Alagesan S., Chandra T., Bhutani K., Finger millet bran supplementation alleviates obesity-induced oxidative stress, inflammation and gut microbial derangements in high-fat diet-fed mice. Br. J. Nutr., 2014, 112, 1447-1458.

39. Nam H., Jung H., Karuppasamy S., Park Y.S., Cho Y.S., Lee J.Y., Seong S.-I., Suh J.G., Anti-diabetic effect of the soybean extract fermented by Bacillus subtilis MORI in $\mathrm{db} / \mathrm{db}$ mice. Food Sci. Biotechnol., 2012, 21, 1669-1676.

40. Préstamo G., Rupérez P., Espinosa-Martos I., Villanueva M.J., Lasunción M.A., The effects of okara on rat growth, cecal fermentation, and serum lipids. Eur. Food Res. Technol., 2007, 225, 925-928.

41. Schroeder N., Marquart L.F., Gallaher D.D., The role of viscosity and fermentability of dietary fibers on satiety-and adiposity-related hormones in rats. Nutrients, 2013, 5, 2093-2113. 
42. Surel O., Couplet B., Influence of the dehydration process on active compounds of okara during its fractionation. J. Sci. Food Agric., 2005, 85, 1343-1349.

43. Teoh S.L., Latiff A.A., Das S., A histological study of the structural changes in the liver of streptozotocin-induced diabetic rats treated with or without Momordica charantia (bitter gourd). Clin. Ter., 2008, 160, 283-286.

44. Tolman K.G., Fonseca V., Dalpiaz A., Tan M.H., Spectrum of liver disease in type 2 diabetes and management of patients with diabetes and liver disease. Diabetes Care, 2007, 30, 734-743.

45. Tucker A.J., Vandermey J.S., Robinson L.E., Graham T.E., Bakovic M., Duncan A.M., Effects of breads of varying carbohydrate quality on postprandial glycaemic, incretin and lipidaemic response after first and second meals in adults with diet-controlled type 2 diabetes. J. Funct. Foods, 2014, 6, 116-125.

46. Villanueva M., Yokoyama W., Hong Y., Barttley G., Rupérez P., Effect of high-fat diets supplemented with okara soybean by-product on lipid profiles of plasma, liver and faeces in syrian hamsters. Food Chem., 2011, 124, 72-79.

47. Xu B.-Q., Yang P., Zhang, Y.-Q., Hypoglycemic activities of lyophilized powder of Gynura divaricata by improving antioxidant potential and insulin signaling in type 2 diabetic mice. Food Nutr. Res., 2015, 59, 29652.

48. Yogo T., Ohashi Kunihiko Terakado Y., Harada Y., Nezu Y., Hara Y., Tagawa M., Kageyama H., Fujisawa T., Influence of dried okara-tempeh on the composition and metabolites of fecal microbiota in dogs. Int. J. Appl. Res. Vet. Med., 2011, 9, 176-183.

49. Zheng J., Shen N., Wang S., Zhao G., Oat beta-glucan ameliorates insulin resistance in mice fed on high-fat and high-fructose diet. Food Nutr. Res., 2013, 57, 22754.

Submitted: 2 July 2016. Revised: 20 September 2016. Accepted: 8 November 2016. Published on-line: 21 April 2017. 
